\title{
Near-real time oculodynamic MRI: a feasibility study for evaluation of diplopia in comparison with clinical testing
}

\author{
Isabelle Berg • Anja Palmowski-Wolfe • Katja Schwenzer-Zimmerer • \\ Cornelia Kober • Ernst-Wilhelm Radue • Hans-Florian Zeilhofer • Klaus Scheffler • \\ Christoph Kunz $\cdot$ Carlos Buitrago-Tellez
}

Received: 6 March 2011 / Accepted: 21 July 2011 /Published online: 14 August 2011

(C) European Society of Radiology 2011

\begin{abstract}
Objective To demonstrate feasibility of near-real-time oculodynamic magnetic resonance imaging (od-MRI) in depicting extraocular muscles and correlate quantitatively the motion degree in comparison with clinical testing in patients with diplopia.

Methods In 30 od-MRIs eye movements were tracked in the horizontal and sagittal plane using a a TrueFISP sequence with high temporal resolution. Three physicians graded the visibility of extraocular muscles by a qualitative scale. In 12 cases, the maximal monocular excursions in the horizontal and vertical direction of both eyes were measured in od-MRIs and a clinical test and correlated by the Pearson test.

Results The medial and lateral rectus muscles were visible in the axial plane in $93 \%$ of the cases. The oblique, superior and inferior rectus muscles were overall only in $14 \%$ visible. Horizontal $(p=0,015)$ and vertical $(p=0,029)$ move-
\end{abstract}

Electronic supplementary material The online version of this article (doi:10.1007/s00330-011-2232-1) contains supplementary material, which is available to authorized users.

I. Berg $\cdot$ K. Schwenzer-Zimmerer $\cdot$ H.-F. Zeilhofer $\cdot$ C. Kunz Hightech Research Center of Cranio-Maxillofacial Surgery and Dept. of Cranio-Maxillofacial Surgery, University Hospital Basel, Basel, Switzerland

\section{A. Palmowski-Wolfe}

Department of Ophthalmology, University of Basel,

Basel, Switzerland

\section{Kober}

Faculty of Life Sciences, HAW Hamburg,

Hamburg, Germany ments of the right eye and vertical movement of the left eye $(p=0,026)$ measured by od-MRI correlated positively to the clinical measurements.

Conclusions Od-MRI is a feasible technique. Visualization of the horizontal/vertical rectus muscles is better than for the superior/inferior oblique muscle. Od-MRI correlates well with clinical testing and may reproduce the extent of eye bulb motility and extraocular muscle structural or functional deteriorations.

Key Points

- Oculodynamic MRI technique helps clinicians to assess eye bulb motility disorders

- MRI evaluation of eye movement provides functional information in cases of diplopia

- Oculodynamic MRI reproduces excursion of extraocular muscles with good correlation with clinical testing

- Dynamic MRI sequence supplements static orbital protocol for evaluation of motility disorders

E.-W. Radue

Department of Neuroradiology, University Hospital Basel,

Basel, Switzerland

K. Scheffler

Division of Radiological Physics, University Hospital Basel,

Basel, Switzerland

C. Buitrago-Tellez $(\bowtie)$

Spital Zofingen AG, Institute of Radiology, Zofingen,

Switzerland and University Hospital Basel,

Hightech Research Center of Cranio-Maxillofacial Surgery,

Mühlethalstr. 27,

4800 Zofingen, Switzerland

e-mail: carlos.buitrago@spitalzofingen.ch 
Keywords Diplopia $\cdot$ Magnetic resonance imaging $\cdot$ Orbital trauma $\cdot$ Extraocular muscles $\cdot$ Orbit

\section{Introduction}

The term diplopia is derived from the Greek wordsdiplous, meaning double, and ops, meaning eye. Reasons for diplopia vary and the field is very broad. From the imaging point of view either unenhanced computed tomography $(\mathrm{CT})$ or magnetic resonance imaging (MRI) can be used to evaluate the orbital area. Computed tomography allows precise assessment of the extent of the bony orbit in the acute setting as well as the adjacent soft tissue structures. However, MRI, is advantageous in the diagnosis of adhesions and herniation of orbital soft tissues. Moreover dynamic information can only be gathered by MRI as CT is not an option due to the dose of ionizing radiation especially on the lens. The muscle paths are well evaluated by static magnetic resonance imaging [1] but additional dynamic information can only be seen in an oculodynamic MRI examination. Advances in technology made it possible to acquire images with a higher temporal resolution. Dynamic nearreal-time MRI has been already used in different clinical settings [2-4]. For orbital lesions, this allows to gather images continuously during normal, continuous eye movements.

The aim of this technical note was to evaluate the eye movements qualitatively and quantitatively using a novel technique without tracking device, near-real-time oculodynamic MRI (od-MRI), in comparison with validated clinical test in a group of patients with ocular motility disorders and healthy volunteers.

\section{Methods}

The study was conducted according to the tenets of the Declaration of Helsinki and approved by the local ethics committee. This study includes 5 od-MRI of 5 healthy volunteers aged between 26 and 48 years as well as 25 odMRIs of patients (22 patients, among them 3 patients underwent 2 oculodynamic MRIs in different follow-up intervals) with diplopia. The 22 patients with diplopia were divided into following 5 groups: Post traumatic: Early diplopia $(n=9)$; post traumatic: Late diplopia $(n=7)$; periorbital tumor $(n=2)$; high myopia with diplopia $(n=2)$; unclear ocular motility disorder $(n=5)$. From a total of 30 od-MRI all of them were included for the qualititative evaluation of the visibility of intraocular muscles during movement. Clinical testing was available in 13 patients, one of them only in the horizontal excursion. Thus, for direct correlation of od-MRI with clinical measurements 12 data sets were used.

The eye movements were recorded in the horizontal, coronal and sagittal plane utilizing 1.5-T MRI (Avanto, Siemens, Erlangen, Germany) and a TrueFISP (balanced SSFP) sequence.

The supine patient's head was placed into a standard 8 channel head coil. A foam head form cushioned the patient's head and added stability. There was no need for using bite bars or other devices to secure the patient's head against unintentional movement.

At first the regular protocol of static MRI sequences was applied (routine MRI protocol), including high resolution $\mathrm{T} 1 \mathrm{w}$ and $\mathrm{T} 2 \mathrm{w}$ images in the axial and coronal plane through the orbit. After the patient was instructed to move the eyes during the imaging sequence (time acquisition max. $30 \mathrm{~s}$ ) maximally in the desired direction, a dynamic TrueFISP sequence (mixed T2/T1weighted contrast, temporal resolution $180 \mathrm{~ms}$ per image, spatial resolution $1.3 \mathrm{~mm} \times 1.3 \mathrm{~mm}$, slice thickness $5 \mathrm{~mm}$, generalized autocalibrating partially parallel acquisitions GRAPPA factor 2) was performed axially at the level of the medial and lateral rectus muscles as well as oblique sagittal at the plane along the optical nerve (Fig. 1).

While running the axial TrueFISP sequence the patients were told to slowly look as far as possible to the right and thereafter to left side for three to four times to record the movement in the horizontal plane. The upto-downward eye movement was recorded in a second, sagittal sequence. The additional MRI time needed for our study was little as each sequence would only take between 15-20 s. The shortest acquisition time took $5 \mathrm{~s}$ for one sequence. After concluding the MR examination, eye movement was assessed in the sagittal, axial and coronal plane utilizing a regular desktop PC and the avi movies of the TrueFISP sequences.

Three experienced physicians (radiologist, ophthalmologist, maxillofacial surgeon) independently evaluated the visibility of the extraocular muscles as follows: Grade 1 (not visualized at all), grade 2 (partially/inadequate visualisation), grade 3 (complete visualisation with minor image quality), grade 4 (complete visualization with good image quality) and grade 5 (completely visualized with very good image quality). For the correlation with od-MRI the patient's files in the 12 cases available were reviewed for the clinical testing results of the eye movement. The monocular horizontal (Fig. 2) and vertical excursions in $\mathrm{mm}$ (clinical testing) were set in relation to the greatest excursion of eye movements on the oculodynamic MRI. 


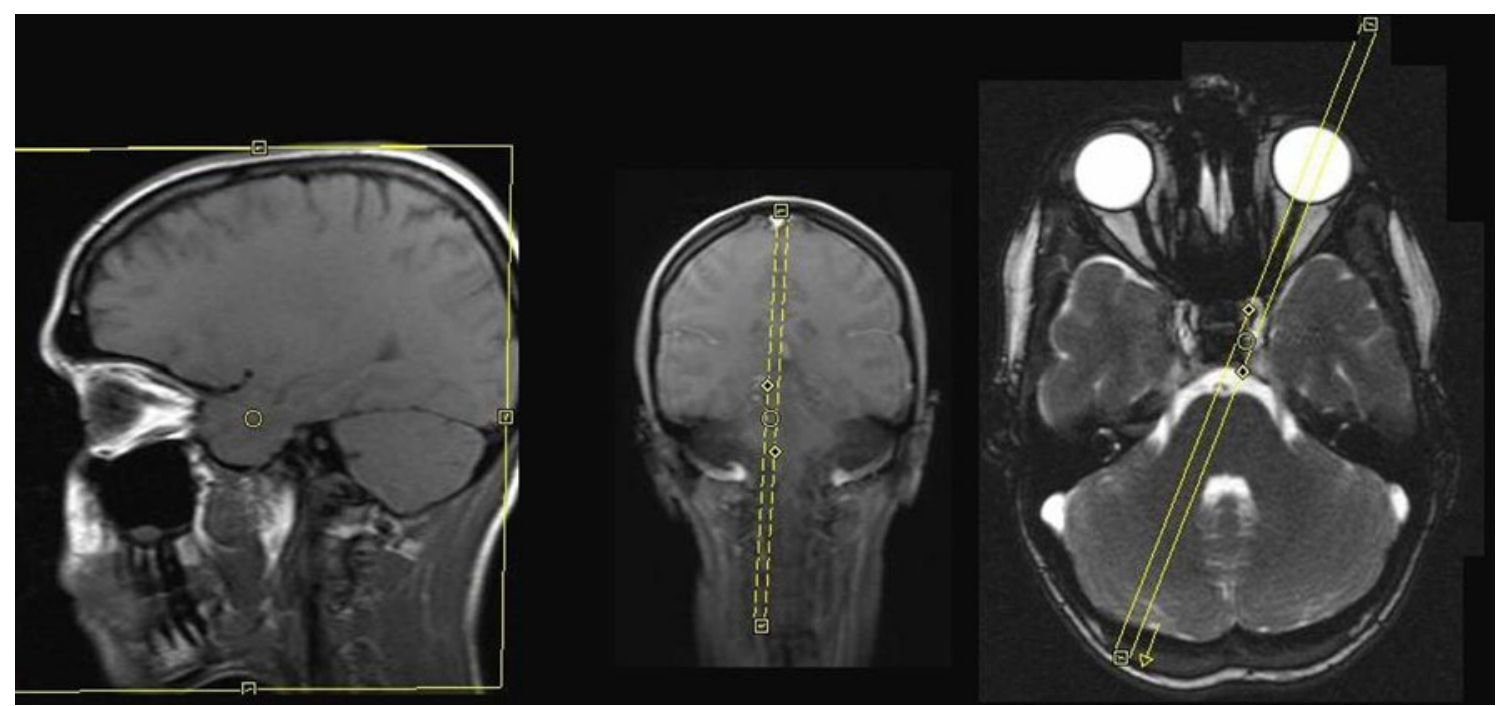

Fig. 1 Planning of MR examination. The yellow square outlines part of the block that will be scanned

In the clinic, ocular motility was assessed according to the Limbus test by Kestenbaum [5]: In primary position, the position of the corneal limbus was noted relative to a ruler held in front of the eye. The patient was then asked to look as far as possible to the right or respectively to the left side, and also as far as possible upwards or downwards. The maximal shift of the corneal limbus was seen on the ruler and noted as an eye movement in $\mathrm{mm}$. The measurement for the oculodynamic MRI was done with two angle

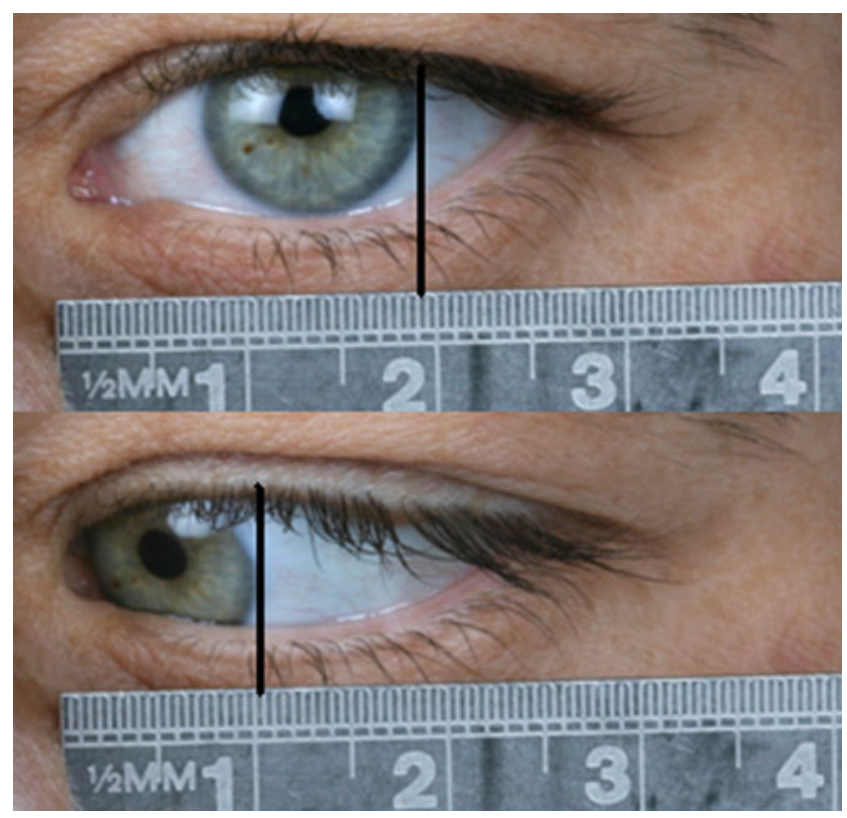

Fig. 2 Clinical measurement of the monocular horizontal excursion: the distance between the two black lines measures $7,5 \mathrm{~mm}$. This is a normal reading for a healthy proband measurements using screen shots from the oculodynamic MRI sequence (Fig. 3).

The reference line was the midline through the sella turcica for the movement to the left and to the right. For the vertical movement the centre of the lens was in maximal upgaze and in maximal downgaze was used in relation to the maximal eye length. It is important to have the lens well identified and before the examination is completed it should be checked if the lens is on the image. Otherwise it is only possible to evaluate the movement subjectively. For the statistical analysis the Pearson's Test and the computer software SPSS version 11.0 was used. Significance was expected below the $p$ value of 0.05 . Using the Pearson's test there was no need to convert eye movement from $\mathrm{mm}$ into degrees for comparison.

\section{Results}

Od-MRI imaging technique with the TrueFISP sequence was robust and constant diagnostic image quality could be obtained. From a total of 30 qualitatively evaluated oculodynamic MRI studies, the medial and lateral rectus muscles were visible in the axial plane in $93 \%$ of the cases. The oblique, superior and inferior rectus muscles were overall only in $14 \%$ adequately visible. It was most difficult to evaluate the superior and inferior oblique muscles while the horizontal and vertical extraocular muscles could be consistently seen well in most of the TrueFISP sequences (Fig. 4).

The optic nerve movement evaluations as well as the orbital bulb motions were well visible documented with high qualitative values (Fig.5). 


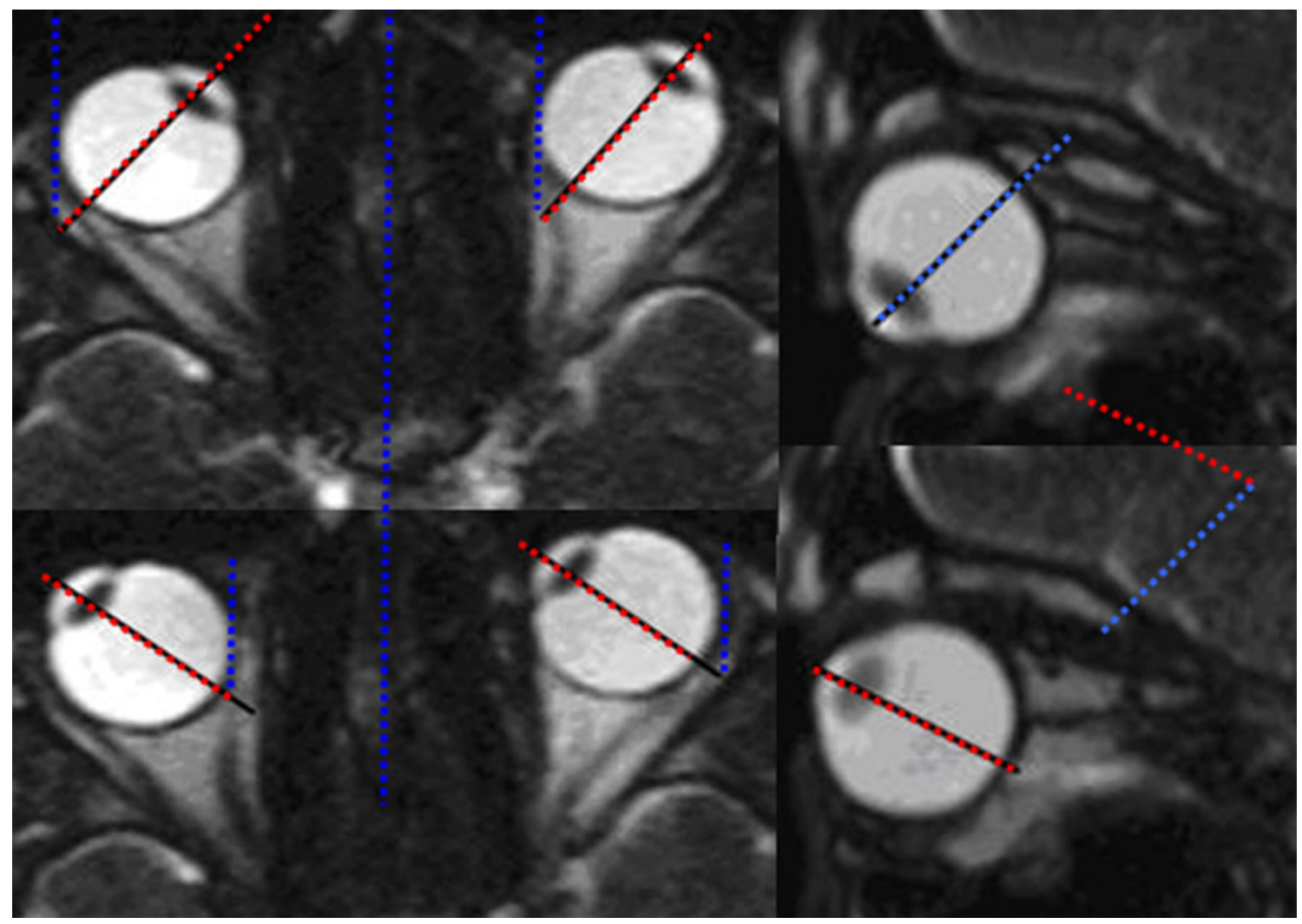

Fig. 3 Single images taken from a dynamic TrueFISP sequence. MRI measurement of the maximal monocular horizontal excursion on the right side of the figure. Blue: sagittal axis. Red: axis of the bulbus oculi. The angle between the red and the blue line renders the maximal excursion of the eye to that side. MRI measurement of the maximal monocular vertical excursion on the left side of the figure. Blue:

In the quantitive evaluation of od-MRI with clinical testing in 12 cases the correlation of the clinical maximal bulbus excursion $(\mathrm{mm})$ correlated significantly with the quantitative measurements by od-MRI (angles) by using the Pearson's Test for the horizontal movement of the right ( $p=$ $0,015)$ and left eye $(p=0,026)$ as well as the vertical movement of the right eye $(p=0,029)$. The vertical movement of the left eye was slightly above the signifi- maximal upward rotation of the bulbus oculi. Red: maximal downward rotation of the blue line renders the maximal vertical movement of bulbus oculi. The angle between the red and the blue line renders the maximal vertical movement of the eye. $222 \times 138 \mathrm{~mm}$ $(96 \times 96 \mathrm{DPI})$

cance level $(p=0,091)$. The complete values are summarized in Table 1 .

In the attached od-MRI movies (Movie 1-sagittal excursion and 2-horizontal excursion) complete visualization of extraocular muscles during movement with good image quality graded with a " 4 " are documented. In movie 3 a patient with post-traumatic diplopia with entrapment of the inferior rectus muscle is demonstrated.

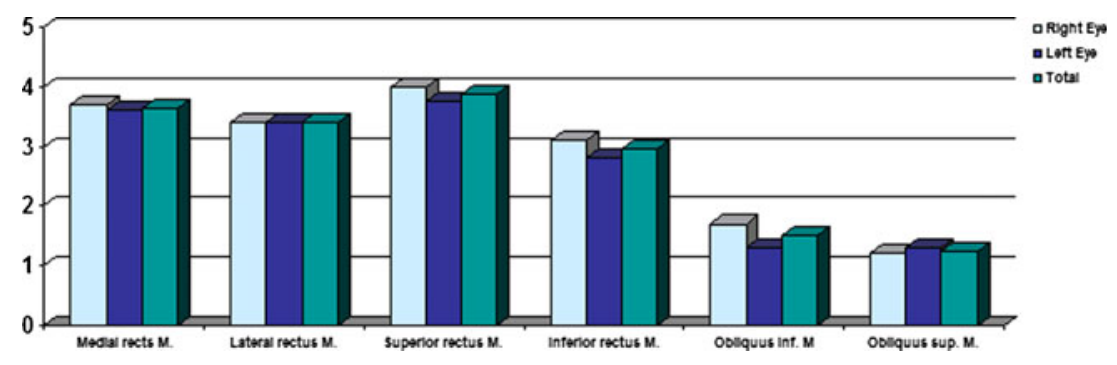

Fig. 4 Visualization of the extraocular muscles: Grade 1 (not visualized at all), grade 2 (partially/ inadequate visualization), grade 3 (complete visualisation with minor image quality), grade 4 (complete visualization with good image quality) and grade 5 (completely visualized with very good image quality) 


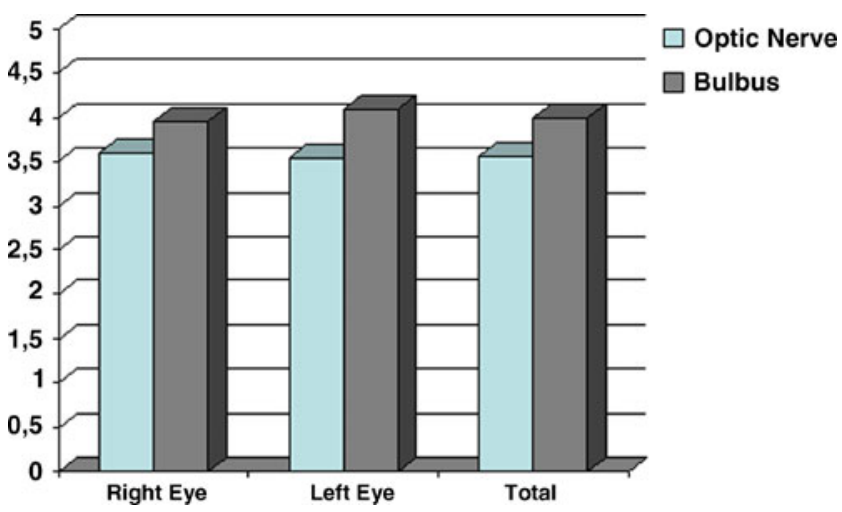

Fig. 5 Visualization of the optic nerve and bulbus: Grade 1 (not visualized at all), grade 2 (partially/ inadequate visualization), grade 3 (complete visualisation with minor image quality), grade 4 (complete visualization with good image quality) and grade 5 (completely visualized with very good image quality)

\section{Discussion}

Diagnostic and therapeutic management of patients with diplopia remains challenging. Up to now, only a few studies used dynamic or cine MRI to investigate eye movement and the movement of the surrounding tissues in particular the extraocular muscles [6-10]. So far cine/near-real-time MRI research was focused on and was developed for temporomandibular joint and swallowing [4, 11-13]. Simultaneously to these endeavours $[4,14]$ some progress was achieved in the field of ocular dynamic MRI. Recent studies used a cine sequence and patients were asked to fixate on single numbered marks [15]. Kau $\mathrm{HC}$ et al. [16] and Piccirelli $\mathrm{M}$ et al. [17] utilized a cine MRI sequence which acquires multiple images in a given time during one single acquisition. On the other hand these approaches needed a tracking system [16] and have taken up to 4-5 min [17] for each image generation.

The near-real-time oculodynamic MR approach used in our study needed no eye tracking device. The functional dynamic sequence was introduced into the clinical routine orbital protocol without any additional devices in cooperating patients after short instruction to the patients prior to scanning. Indeed, the missing tracking technique may influence the whole range of possible eye movements observed. In fact, it might be the case that the free method may reproduce real maximal excursion more realistically. Overall acquisition time is very short as it almost operates in "near real time". Each of our recordings lasted about 15- 20 s. A potential clinical application for this sequence is an early detection of mechanically induced motility disorders for instance of the eye due to trauma and/or after surgical intervention. The use of the od-MRI in long term follow up for patients with orbital injuries seems to be acceptable in terms of limiting radiation dose to the patient. Minor susceptibility artefacts might blind the assessment of the titanium/periorbital tissue interface.

Another limitation might be the in-plane resolution of the oculodynamic sequence. While at present $1.3 \mathrm{~mm}$ in-plane resolution can be achieved with cine sequences, conventional static orbital MRI imaging can render a resolution of down to an intraorbital resolution of 234- $312 \mu \mathrm{m}$ within $1.5-$ to $2.0-\mathrm{mm}$ thick planes [18]. A comparative study with the goal in how many cases the dynamic MRI could completely replace the time consuming static MRI to answer the question of the source of double vision might be interesting. However, the need for high resolution images from static MRI orbital protocols is not replaced by od-MRI. We see the potential added value of this technical development in a more integrating and supplementing practical way of adding functional information.

In conclusion, to our knowledge, there are no other studies reported which assess and correlate quantitatively oculodynamic MRI with a clinical testing. The near real-time od- MRI correlates well with clinical testing and might be a potentially better practical tool to reproduce the extent of eye bulb motility and extraocular muscle structural or functional deteriorations than static orbital MRI. Furthermore, the practical use of this new technique is feasible, well reproducible and implemented in routine orbital MRI protocols without additional devices.

Table 1 Significance level $(p=0,05)$ of correlations between clinical testing (distance in $\mathrm{mm})$ and MRI evaluation (Angle in degree)

\begin{tabular}{lll}
\hline Correlation between & $\begin{array}{l}\text { Significance } \\
\text { (2-sided) }\end{array}$ & $\begin{array}{l}\text { Pearson correlation } \\
\text { coefficient }\end{array}$ \\
\hline Horizontal movements of the right eye (adduction and abduction) $\rightarrow$ clinical and MRI & 0,015 & 0,681 \\
Horizontal movements of the left eye (adduction and abduction) $\rightarrow$ clinical and MRI & 0,026 & 0,636 \\
Vertical movement of the right eye (upwards and downwards) $\rightarrow$ clinical and MRI & 0,029 & 0,595 \\
Vertical movement of the left eye (upwards and downwards) $\rightarrow$ clinical and MRI & 0,091 & 0,684 \\
\hline
\end{tabular}


Acknowledgements Christoph Kunz and Carlos Buitrago-Tellez contributed equally to this work. Special thanks to the MRI radiological technician team of the Department of Radiology of the University of Basel, Switzerland for valuable support for image acquisition

\section{References}

1. Kono R, Clark RA, Demer JL (2002) Active pulleys: magnetic resonance imaging of rectus muscle paths in tertiary gazes. Invest Ophthalmol Vis Sci 43:2179-2188

2. Palmowski-Wolfe AM, Kober C, Berg I, Kunz C, Wetzel S, Buitrago-Téllez C, Radü EW, Scheffler K (2009) Globe restriction in a severely myopic patient visualized through oculodynamic magnetic resonance imaging (od-MRI). J AAPOS 13:322-4

3. Palmowski-Wolfe AM, Berg I, Wetzel S, Kunz C, Radü EW, Scheffler K, Buitrago-Téllez C, Kober C (2010) Bilateral VI nerve injury. Ophthalmology 117:398.e1-2

4. Abolmaali ND, Schmitt J, Schwarz W, Toll DE, Hinterwimmer S, Vogl TJ (2004) Visualization of the articular disk of the temporomandibular joint in near-real-time MRI: feasability study. Eur Radiol 14:1889-94

5. Rüssmann W (2003) Basic methods of strabismology. Ophthalmologe 100:416-30, quiz 431-2

6. Bailey CC, Kabala J, Laitt R, Weston M, Goddard P, Hoh HB, Potts MJ, Harrad RA (1993) Cine magnetic resonance imaging of eye movements. Eye (Lond) 7(Pt 5):691-693

7. Shin GS, Demer JL, Rosenbaum AL (1996) High resolution, dynamic, magnetic resonance imaging in complicated strabismus. J Pediatr Ophthalmol Strabismus 33:282-90

8. Speeg-Schatz C (2002) MRI used in the exploration of occulomotor muscles. J Fr Ophtalmol 25:956-8

9. Eter N, Garbe S, Pauleit D, Schüttoff T, Schüller H (2003) Magnetic resonance imaging analysis of anterior and posterior eye segment displacement during ocular gaze shifts. Eur J Ophthalmol 13:196-201

10. Krzizoh TH, Kaufmann H, Traupe H (1997) Elucidation of restrictive motility in high myopia by magnetic resonance imaging. Arch Ophthalmol 115:1019-27

11. Katzberg RW, Bessette RW, Tallents RH, Plewes DB, Manzione JV, Schenck JF, Foster TH, Hart HR (1986) Normal and abnormal temporomandibular joint: MR imaging with surface coil. Radiology 158:183-189

12. Burnett KR, Davis CL, Read J (1987) Dynamic display of the temporomandibular joint meniscus by using "fastscan" MR imaging. Am J Roentgenol 149:959-962

13. Barkhausen J, Goyen M, von Winterfeld F, Lauenstein $T$, Arweiler-Harbeck D, Debatin JF (2002) Visualization of swallowing using real-time TrueFISP MR fluoroscopy. Eur Radiol 12:129-33

14. Hayakawa Y, Kober C, Otonari-Yamamoto M, Otonari T, Wakoh M, Sano T (2007) An approach for three-dimensional visualization using high-resolution MRI of the temporomandibular joint. Dentomaxillofac Radiol 36:341-347

15. Kakizaki H, Selva D, Leibovitch I (2010) Dynamic study of the medial and lateral recti capsulopalpebral fasciae using cine mode magnetic resonance imaging. Ophthalmology 117:388-91

16. Kau HC, Tsai CC, Ortube MC, Demer JL (2007) High-resolution magnetic resonance imaging of the extraocular muscles and nerves demonstrates various etiologies of third nerve palsy. Am J Ophthalmol 143:280-287

17. Piccirelli M, Luechinger R, Rutz AK et al (2007) Extraocular muscle deformation assessed by motion-encoded MRI during eye movement in healthy subjects. J Vis 7:5.1-10

18. Demer JL, Ortube MC, Engle EC, Thacker N (2006) Highresolution magnetic resonance imaging demonstrates abnormalities of motor nerves and extraocular muscles in patients with neuropathic strabismus. J AAPOS 10:135-42 